\title{
Diseño basado en prestaciones de observadores PI para el diagnóstico de fallos en sistemas lineales con perturbaciones gaussianas
}

\author{
Ester Sales-Setién, Ignacio Peñarrocha, Daniel Dolz y Roberto Sanchis \\ Departamento de Ingeniería de Sistemas Industriales y Diseño \\ Universitat Jaume I, Castellón, España \\ \{esales,ipenarro,ddolz,rsanchis\}@uji.es
}

\section{Resumen}

Este trabajo aborda el problema de diagnóstico de fallos en sistemas lineales e invariantes en el tiempo bajo la presencia de perturbaciones gaussianas, a través de observadores basados en modelo de tipo proporcional-integral con ganancias predefinidas. Para ello, se propone un diseño conjunto de los generadores y evaluadores de residuos de fallo teniendo en cuenta el compromiso entre parámetros físicos como la tasa de falsas alarmas, los mínimos fallos detectables, y la integral del cuadrado del error de los residuos ante fallos escalón. También se aborda el problema de aislamiento dinámico de fallos. Para resolver este diseño se presentan dos enfoques distintos: el primero basado en el filtro de Kalman de régimen permanente y el segundo basado en técnicas de optimización convexa.

Palabras clave: diagnóstico de fallos, observador PI, filtro de Kalman, BMIs.

\section{INTRODUCCIÓN}

El diagnóstico de fallos ha recibido mucha atención en las últimas décadas $[1,14]$. Un sistema de diagnóstico de fallos incluye tres tareas principales: detección, aislamiento y análisis de fallos (FDIA). Mientras que la detección de fallos (FD) consiste en determinar la aparición de un fallo en el sistema, el aislamiento de fallos (FI) se centra en su localización y el análisis de fallos (FA) trata de caracterizar la naturaleza y magnitud del mismo [9]. Para el diseño de los diagnositcadores, las técnicas basadas en modelo han sido ampliamente estudiadas.

En general, un sistema FDIA está formado por generadores y evaluadores de residuos de fallos. Entre las estrategias de generación de residuos basadas en modelo, hay una tendencia creciente en el uso observadores, ver $[13,15,27]$ y las referencias allí incluidas. Teniendo en cuenta la dualidad entre controlabilidad y observabilidad, la disminución del error en régimen permanente debida al término integral de los controladores ha motivado la incorporación de un término integral en el diseño de observadores de fallo. Este tipo de obser- vadores proporcionales-integrales (PI) se propone en $[7,20,23]$ donde el uso de un estado aumentado posibilita la FDIA. En lo referente a la evaluación de los residuos, las estrategias más utilizadas para conseguir un tratamiento óptimo de los residuos generados por los observadores [4] son los métodos estadísticos y las técnicas basadas en normas. Tal y como se afirma en [5], el diseño conjunto de los generadores y evaluadores de residuos de fallo es la clave para conseguir un sistema FDIA óptimo.

El primer problema a tener en cuenta en el diseño de sistemas FDIA es la presencia de entradas desconocidas $[21,22,25]$. Actualmente, el diseño robusto de FDIA es un área muy activa. Hay dos enfoques principales para atacar este problema. El primero, usado en $[2,24]$, propone eliminar completamente el efecto de las perturbaciones desconocidas en los residuos. Cuando un desacoplamiento total no es posible, el efecto de esas perturbaciones debería minimizarse [3,30]. Sin embargo, no basta con que los residuos sean robustos ante entradas desconocidas, sino que deben ser tan sensibles como sea posible a los fallos $[11,29]$. En el enfoque basado en normas, Zhang y Ding [28] proponen buscar un compromiso entre la tasa de falsas alarmas (FAR) y la tasa de fallos detectados (FDR) para conseguir el comportamiento requerido del diagnosticador de fallos. Sin embargo, esos parámetros no dan información sobre otras cuestiones importantes, como el tamaño de los fallos que pueden ser detectados, el comportamiento dinámico de los residuos para su aislamiento o la precisión en régimen permanente para la identificación del fallo. Los diseños basados en optimización $[6,26]$ incluyen los fallos mínimos que deben detectarse y una cota conservadora del FAR por medio de la desigualdad de Markov. En esos trabajos, suponiendo perturbaciones gaussianas, el FAR es posteriormente acotado con más precisión por medio de procedimientos iterativos sobre el problema de optimización inicial. En [6], la capacidad de seguimiento se incluye en el diseño por medio de un ratio de decaimiento que implica una restricción adicional. Este conservadurismo puede reducirse expresando el comportamiento dinámico de los residuos en función de la integral del error al cuadrado (ISE) [26]. 
En este trabajo se propone una estrategia FDIA centrada en tareas de aislamiento, que busca un compromiso entre la precisión y capacidad de seguimiento del diagnosticador a través del uso de parámetros con significado físico que tienen en cuenta todos los aspectos anteriores. Así, se presenta un método de diseño conjunto de los generadores y evaluadores del residuo, que incluye explícitamente cotas ajustadas del FAR bajo ruidos gaussianos, la magnitud de los fallos mínimos detectables, el ISE del residuo frente a fallos en escalón y una medida del aislamiento dinámico de fallos. Sin la necesidad de recurrir a procedimientos iterativos, se presentan dos propuestas de diseño simples que se basan en observadores PI con ganancias constantes. La primera técnica se basa en el filtro de Kalman de régimen permanente que se resuelve mediante algoritmos de optimización heurísticos. El segundo método es un enfoque basado en normas, que se formula a través de desigualdades matriciales bilineales (BMI) y se resuelven mediante optimización convexa iterativa.

El trabajo se estructura de la siguiente forma. En primer lugar se describe el problema en la Sección 2 , donde se define el modelo matemático de los sistemas considerados, se presenta la estrategia propuesta de generación y evaluación del residuo y se definen los parámetros que definen las prestaciones consideradas en el diseño. En la Sección 3, se muestra como incluir esas prestaciones en las dos propuestas de diseño del sistema FDIA. En la Sección 4 se validan las estrategias propuestas por medio de simulaciones. Finalmente, en la Sección 5 se resumen las conclusiones.

Notación: Sea $M$ una matriz cuadrada de tamaño $n \times n$. $M(i, i)$ representa el elemento diagonal $i$-ésimo de la matriz $M$, mientras $M(i)$ representa la $i$-esima fila o columna, dependiendo del contexto. $M \succeq 0$ significa que $M$ es semidefinida positiva ( $\preceq$ para semidefinida negativa). La suma directa se representa como $\bigoplus$. Sea $x_{k} \in \mathbb{R}^{n}$ una señal temporal discreta. $x_{k}(i)$ representa el elemento $i$-ésimo del vector $x_{k}$. Se escribe $\left\|x_{k}\right\|_{2}^{2} \triangleq x_{k}^{T} x_{k}$ para la norma $l_{2}$ de $x_{k}$ y $\left\|x_{k}\right\|_{R M S}^{2} \triangleq \lim _{K \rightarrow \infty} \sum_{k=0}^{K-1} \frac{1}{K}\left\|x_{k}\right\|_{2}^{2}$ para la norma RMS de $x_{k}$. El valor esperado y la probabilidad se representan como $\mathbf{E}\{\cdot\}$ y $\operatorname{Pr}\{\cdot\}$.

\section{DESCRIPCIÓN DEL PROBLEMA}

\subsection{Modelo en representación interna}

Considérese un sistema lineal discreto invariante en el tiempo (LTI) definido por las ecuaciones

$$
\begin{aligned}
x_{k+1} & =A x_{k}+B u_{k}+E f_{k}+G v_{k}, \\
y_{k} & =C x_{k}+D u_{k}+F f_{k}+H v_{k},
\end{aligned}
$$

donde $x \in \mathbb{R}^{n_{x}}$ es el vector de estado, $y \in \mathbb{R}^{n_{y}}$ es el vector de salida, $u \in \mathbb{R}^{n_{u}}$ es el vector de entrada. El vector $v \in \mathbb{R}^{n_{v}}$ incluye ruidos gaussianos de media nula y covarianza conocida $\mathbf{E}\left\{v_{k} v_{k}^{T}\right\}=V$. Finalmente, el vector $f \in \mathbb{R}^{n_{f}}$ incluye los fallos que afectan al sistema. Se supone que todos los fallos son detectables, aislables e identificables ${ }^{1}$.

Para cumplir con los objetivos del diagnóstico de fallos, se propone un esquema que utiliza observadores PI basados en modelo. Por tanto, se modelan los fallos $f_{k}$ de (1) como señales de variación lenta, es decir,

$$
f_{k+1}=f_{k}+\Delta f_{k}
$$

donde $\Delta f_{k}$ representa la variación de la señal de fallo entre instantes consecutivos. La ecuación (2) permite modelar, por ejemplo, señales escalón ( $\Delta f_{k}$ solo toma valores no nulos en el instante de aparición del fallo), or señales tipo rampa $\left(\Delta f_{k}\right.$ toma un valor constante). Este tipo de modelo de fallo se utiliza en la literatura con frecuencia para analizar el comportamiento de algoritmos de diagnóstico de fallos, por ejemplo en $[17,19]$. Para incluir la dinámica de los fallos, se utiliza un modelo extendido del sistema (1)

$$
\begin{aligned}
z_{k+1} & =\bar{A} z_{k}+\bar{B} u_{k}+\bar{G} v_{k}+\bar{E} \Delta f_{k} \\
y_{k} & =\bar{C} z_{k}+\bar{D} u_{k}+\bar{H} v_{k}
\end{aligned}
$$

donde $z_{k}=\left[\begin{array}{ll}x_{k}^{T} & f_{k}^{T}\end{array}\right]^{T}$ es el vector de estado extendido,

$$
\begin{gathered}
\bar{A}=\left[\begin{array}{cc}
A & E \\
0 & I
\end{array}\right], \bar{B}=\left[\begin{array}{c}
B \\
0
\end{array}\right], \bar{G}=\left[\begin{array}{c}
G \\
0
\end{array}\right], \bar{E}=\left[\begin{array}{l}
0 \\
I
\end{array}\right], \\
\bar{C}=\left[\begin{array}{ll}
C & F
\end{array}\right], \bar{D}=D \text { and } \bar{H}=H .
\end{gathered}
$$

\footnotetext{
${ }^{1}$ Definimos la matriz de transferencia entre un fallo $f(i)$ y la salida como $G_{f(i)}[z]=C(z I-A)^{-1} E(i)+$ $F(i)$. Tal y como se demuestra en [4], un fallo $f(i)$ en un sistema de la forma (1) es detectable si y solo si $G_{f(i)}[z] \neq 0$, es aislable si y solo si

$$
\operatorname{rank}\left[G_{f(1)}[z] \ldots G_{f\left(n_{f}\right)}[z]\right]=\sum_{i=1}^{n_{f}} \operatorname{rank}\left(G_{f(i)}[z]\right),
$$

y finalmente, es identificable si y solo si la inversa de la matriz de transferencia $G_{f(i)}[z]$ es estable.
} 


\subsection{Generación y Evaluación del Residuo}

Nuestra propuesta consiste en resolver el problema FDI utilizando técnicas de identificación del fallo; por tanto se definen $n_{f}$ residuos $r(i)$ como las estimaciones de $f(i)$, es decir, $r(i):=\hat{f}(i)$. Para generar esos residuos, se propone el siguiente observador PI con ganancia predefinida, basado en el modelo extendido (3):

$$
\begin{aligned}
\hat{z}_{k+1} & =\bar{A} \hat{z}_{k}+\bar{B} u_{k}+L\left(y_{k}-\bar{C} \hat{z}_{k}-\bar{D} u_{k}\right), \\
r_{k} & =R \hat{z}_{k}
\end{aligned}
$$

donde $L$ es la matriz de ganancias que actualiza con las mediciones la predicción obtenida a partir del modelo. $R$ es la matriz que extrae los fallos del vector de estado extendido $z_{k}$, es decir, $f_{k}=R z_{k}$ con $R=\left[\begin{array}{ll}0_{n_{f} \times n_{x}} & I_{n_{f} \times n_{f}}\end{array}\right]$. Nótese que se define $R(i)$ como la $i$-ésima fila de $R$, por lo que un fallo se extrae como $f_{k}(i)=R(i) z_{k}$. Se define el error de estimación asociado a los residuos como

$$
e_{k}=f_{k}-r_{k}
$$

cuya dinámica viene dada por

$$
\begin{aligned}
\tilde{z}_{k+1} & =(\bar{A}-L \bar{C}) \tilde{z}_{k}+(\bar{G}-L \bar{H}) v_{k}+\bar{E} \Delta f_{k} \\
e_{k} & =R \tilde{z}_{k}
\end{aligned}
$$

con $\tilde{z}_{k}=z_{k}-\hat{z}_{k}$. Definimos $Q$ como la covarianza del error $e_{k}$, es decir, $Q=\mathbf{E}\left\{e_{k} e_{k}^{T}\right\}$.

Nota 1 En ausencia de fallos y suponiendo nulas las condiciones iniciales, el error de estimación $e_{k}$ sigue una distribución de proBabilidad normal multivarible de media nula y covarianza $Q$, ya que las perturbaciones y ruidos de medida siguen una distribución normal de media cero. El error asociado a cada residuo, $e_{k}(i)$, sigue una distribución normal de media cero y varianza $Q(i, i)$, lo que se corresponde con la distribución marginal del elemento en el vector de errores.

Para la evaluación del residuo se define el siguiente criterio de decisión, que permite el aislamiento del fallo:

$$
\begin{cases}\text { if }\left|r_{k}(i)\right| \geq r_{t h}(i) & \text { Fallo } f(i) \\ \text { otro } & \text { No fallo } f(i)\end{cases}
$$

donde $r_{t h}(i)$ es el umbral de residuo asociado al fallo $f(i)$. Se concluye que hay un fallo en el sistema (detección de fallo) si alguna de las alarmas de fallo anteriores, $f(i)$, está activa.

Nota 2 Suponiendo la ausencia de fallos simultáneos, el hecho de detectar y aislar el fallo mediante el mismo mecanismo de decisión (7) no introduce ningún conservadurismo. Este trabajo se centra en fijar las prestaciones del diagnositcador en base a tareas de aislamiento de fallos.

\subsection{Prestaciones del diagnóstico de fallos}

De acuerdo con [1], se definen varios parámetros para caracterizar las prestaciones del diagnosticador de fallos. En primer lugar, la tasa de falsas alarmas (FAR) en el aislamiento de un fallo, que definimos como $\phi(i)$, es la probabilidad de que ocurran falsas alarmas, es decir,

$$
\phi(i)=\operatorname{Pr}\left\{\left|r_{k}(i)\right| \geq r_{t h}(i): f_{k}(i)=0\right\} .
$$

En segundo lugar, el fallo mínimo aislable $f(i)$, que denotamos como $f_{m}(i)$, es el fallo mínimo constante que produce una alarma del fallo $f(i)$ suponiendo que no hay otros fallos o perturbaciones en el sistema, es decir,

$$
f_{m}(i)=\left\{\left|f_{k}(i)\right|: \begin{array}{l}
f_{k}(i)=f_{k-1}(i), \forall k \\
\operatorname{lím}_{k \rightarrow \infty}\left|r_{k}(i)\right| \geq r_{t h}(i)
\end{array}\right\}
$$

En este trabajo, la definición previa implica que $r_{t h}(i)=f_{m}(i)$, porque los residuos se han definido como $r(i):=\hat{f}(i)$.

Nota 3 Si las condiciones iniciales son nulas y en ausencia de fallos, el error de estimación asociado a cada residuo, $e_{k}(i)$, tiene una distribución normal de media cero. Teniendo en cuenta que $r_{t h}(i)=f_{m}(i)$, el fallo minimo aislable puede interpretarse como el cuantil usado para construir el intervalo de confianza $1-\phi(i) / 2$. Así pues, se cumple la siguiente relación

$$
f_{m}(i)=\Phi^{-1}(1-\phi(i) / 2) Q(i, i)^{1 / 2}
$$

donde $\Phi^{-1}$ representa la distribución acumulativa inversa de una variable normal.

En tercer lugar, se define la integral del error al cuadrado (ISE) de un residuo $r(i)$, que denotamos como $\varphi(i)$, como el error de estimación acumulado, es decir,

$$
\varphi(i)=\sum_{k=1}^{\infty} e_{k}^{2}(i)
$$

Mientras que el FAR y los fallos mínimos detectables definen la precisión del diagnosticador de fallos, el ISE de los residuos refleja su capacidad de seguimiento. Zhang y Ding demostraron que existe un compromiso entre el FAR y el FDR [28]. De forma similar, [26] muestra que hay un compromiso entre el FAR, los fallos mínimos detectables y el ISE de los residuos. El objetivo de este trabajo es diseñar generadores y evaluadores del residuo que garanticen cierta FAR y fallo mínimo detectable, a la vez que se maximiza la capacidad de seguimiento (se minimiza el ISE). 


\section{DISEÑO DEL DIAGNOSTICADOR DE FALLOS}

Se proponen dos enfoques para el diseño del diagnosticador de fallos. El primero se basa en u filtro de Kalman, que incluye algunos parámetros de ajuste numéricos sin un significado físico claro, lo que dificulta la selección de los valores más adecuados. En segundo lugar se desarrolla una estrategia basada en BMI que incluye de forma explícita parámetros de ajuste con sentido físico, fáciles de entender, y que se resuelve por medio de una secuencia de problemas de optimización convexa.

\subsection{Diagnóstico de Fallos basado en Filtro de Kalman}

El siguiente teorema muestra como calcular la ganancia constante del observador (4) para el sistema (3) basándose en el filtro de Kalman de régimen permanente.

Teorema 1 Supóngase que $\Delta f_{k}$ tiene una matriz de covarianzas constante y conocida $\Gamma^{-1}$, es decir, $\Gamma^{-1}=\boldsymbol{E}\left\{\Delta f_{k} \Delta f_{k}^{T}\right\}$. La ganancia óptima de Kalman del observador (4) para el sistema (3) en régimen permanente viene dada por:

$$
L=\bar{A} P \bar{C}^{T} \mathcal{C}^{-1}
$$

con $\mathcal{C}=\left(\bar{C} P \bar{C}^{T}+\bar{H} V \bar{H}^{T}\right)$ yP la matriz de covarianzas del vector $\tilde{z}_{k}$, i.e. $P=\boldsymbol{E}\left\{\tilde{z}_{k} \tilde{z}_{k}^{T}\right\}$, que satisface la siguiente ecuación de Ricatti:

$$
\begin{aligned}
& P=\bar{A} P \bar{A}^{T}+\mathcal{G} \mathcal{V} \mathcal{G}^{T}+\bar{A} P \bar{C}^{T} \mathcal{C}^{-1} \bar{C} P \bar{A}^{T}, \\
& \text { con } \mathcal{G}=\bar{G} \oplus \bar{E} \text { and } \mathcal{V}=V \oplus \Gamma^{-1} .
\end{aligned}
$$

Demostración 1 Ver [16].

El resultado anterior permite obtener una ganancia $L$ que estabiliza el observador y tiene en cuenta la presencia de fallos en el sistema. Con la ganancia obtenida, $L$, se puede calcular la covarianza del error de observación en régimen permanente, en el caso de que no haya fallos, resolviendo la siguiente ecuación de Lyapunov:

$$
\begin{aligned}
P_{0} & =(\bar{A}-L \bar{C}) P_{0}(\bar{A}-L \bar{C})^{T} \\
& +(\bar{G}-L \bar{H}) \mathcal{V}(\bar{G}-L \bar{H})^{T}, \\
Q & =R P_{0} R^{T} .
\end{aligned}
$$

Como los fallos no son señales gaussianas, la matriz $\Gamma$ requerida en el Teorema 1 es variante y desconocida, pero puede interpretarse como un parámetro de ajuste cuyo valor puede ser elegido por el diseñador para modificar el comportamiento del diagnosticador de fallos. De esa forma, si se incrementa $\Gamma$ mejora la precisión de los residuos (ya que el filtro se centra más en rechazar los ruidos definidos por $V$ ), mientras que si se reduce $\Gamma$ mejora la capacidad de seguimiento a costa de un menor rechazo del ruido de medida. Para un FAR determinado, una mejor precisión de los residuos implica un valor menor del fallo mínimo detectable, tal y como se deduce de (10).

Un procedimiento para diseñar el diagnosticador de fallos consiste en buscar el valor de $\Gamma$ que da lugar a una matriz $L$ (por medio de (12) y (13)) tal que $Q$ en (14) satisface algunas especificaciones. La siguiente estrategia muestra como diseñar el diagnosticador de fallos basado en filtro de Kalman garantizando unas determinadas tasas de falsa alarma y fallo mínimo detectable mientras se maximiza la capacidad de seguimiento de los residuos.

Estrategia 1 Sea una matriz diagonal $\mathcal{Q}$ definida como

$$
\mathcal{Q}=\bigoplus_{i} \bar{f}_{m}(i)^{2} / \Phi^{-1}(1-\bar{\phi}(i) / 2)^{2}
$$

donde $\bar{\phi}(i)$ representa la tasa de falsas alarmas deseada y $\bar{f}_{m}(i)$ los fallos mínimos detectables deseados. El problema de optimización

$$
\begin{array}{ll}
\operatorname{minimize} & \gamma \\
\text { subject to } & \mathcal{X}_{1}=\left\{\begin{array}{l}
(12),(13),(14), \Gamma \succeq 0, \\
Q \leq \mathcal{Q}, \operatorname{tr}(\Gamma) \preceq \gamma
\end{array}\right\}
\end{array}
$$

a lo largo de las variables $P, P_{0}, Q, L$ y $\Gamma$ da lugar a los generadores de residuos con la respuesta más rápida a los fallos y que garantiza las prestaciones requeridas cuando los umbrales de los evaluadores de los residuos se definen como $r_{t h}(i)=\bar{f}_{m}(i)$. La matriz $Q$ representa la covarianza de los residuos en régimen permanente cuando no hay fallos y queda definida por (14).

El problema de minimización anterior puede resolverse mediante técnicas de optimización heurística como por ejemplo algoritmos genéticos.

\subsection{Diagnóstico de fallos basado en BMI}

El siguiente teorema muestra cómo se puede diseñar la ganancia del observador, $L$, teniendo en cuenta los parámetros de comportamiento requeridos del diagnosticador de fallos.

Teorema 2 Considérese el generador de residuos (4) aplicado al sistema (3). Si existe una matriz L, matrices simétricas $S, \bar{Q}$ y $n_{f}$ matrices simétricas $P_{i}$ y $\Gamma_{i}$ tales que se cumple la condición

$$
\left[\begin{array}{ccc}
S & S \mathcal{A} & S \mathcal{G} V \\
\star & S & 0 \\
\star & \star & V
\end{array}\right] \succeq 0,\left[\begin{array}{cc}
\bar{Q} & R \\
\star & S
\end{array}\right] \succeq 0,
$$


y las siguientes restricciones para $i=\left\{1 \ldots n_{f}\right\}$

$$
\left[\begin{array}{ccc}
P_{i} & P_{i} \mathcal{A} & 0 \\
\star & P_{i} & R(i)^{T} \\
\star & \star & I
\end{array}\right] \succeq 0,\left[\begin{array}{cc}
P_{i} & P_{i} \bar{E} \\
\star & \Gamma_{i}
\end{array}\right] \succeq 0
$$

con $\mathcal{A}=\bar{A}-L \bar{C}$ and $\mathcal{G}=\bar{G}-L \bar{H}$, entonces se cumplen las siguientes afirmaciones:

- En ausencia de fallos y ruidos (es decir, $\left.\Delta f_{k}=v_{k}=0\right)$, el error (5) converge a cero.

- En ausencia de ruidos (es decir, $\left.v_{k}=0\right)$, el ISE de un residuo $r(i)$ ( $\left.i=\left\{1, \ldots, n_{f}\right\}\right)$ debido a un fallo único en forma de escalón unitario $f(j)$ con $j=\left\{1, \ldots, n_{f}\right\}$ está acotado como $\varphi(i) \leq \Gamma_{i}(j, j)$.

- En ausencia de fallos (es decir, $\Delta f_{k}=0$ ), la covarianza del error (5), está acotada como $Q \preceq \bar{Q}$.

Demostración 2 A continuación se demuestran las conclusiones del teorema2.

- Se define la función de Lyapunov $V_{k}^{S}=\tilde{z}_{k} S \tilde{z}_{k}^{T} \quad$ en $\quad$ cada instante $k$. En ausencia de ruidos de medida y fallos, tomando complementos de Schur en la primera desigualdad lineal matricial (LMI) presentada en (17) y premultiplicando el resultado por $\tilde{z}_{k}^{T}$ y postmultiplicando por la traspuesta, se obtiene que $V_{k+1}^{S}-V_{k}^{S} \leq 0$, lo que asegura que el error de estimación del estado (5) converge a cero. Se obtiene el mismo resultado si se define la función de Lyapunov $V_{k}^{P_{i}}=\tilde{z}_{k} P_{i} \tilde{z}_{k}^{T}$ en cada instante $k$ y se opera en la primera LMI presentada en (18).

- realizando operaciones similares en cada una de las siguientes LMIs presentadas en (18) (complementos de Schur y operaciones con $\Delta f_{k}^{T}$ ), tomando valores esperados en el resultado y sumando la restricción obtenida a la anterior, se obtiene

$\boldsymbol{E}\left\{V_{k+1}^{P_{i}}\right\}-\boldsymbol{E}\left\{V_{k}^{P_{i}}\right\}+\boldsymbol{E}\left\{e_{k}^{2}(i)\right\} \leq \Delta f_{k}^{T} \Gamma_{i} \Delta_{k}$

donde se ha tenido en cuenta que las señales $\tilde{z}_{k}$ y $\Delta f_{k}$ no están correladas. Considerando condiciones iniciales nulas y sumando el resultado desde $k=0$ a $K-1$ y tomando el límite cuando $K \rightarrow \infty$, se obtiene $\sum_{k=0}^{\infty} e_{k}^{2}(i) \leq \sum_{k=0}^{\infty} \Delta f_{k}^{T} \Gamma_{i} \Delta f_{k}$. Si una única señal de fallo $f(j)$ cambia en forma de escalón (es decir, $\Delta f_{k}(j)=1$ solo si $k=0$ ), se tiene $\sum_{k=0}^{\infty} e_{k}^{2}(i) \leq \Gamma_{i}(j, j)$. Entonces, acotando los elementos de la diagonal de $\Gamma_{i}$ se limita el ISE de cada uno de los residuos debido a la aparición de cada fallo, lo que demuestra la segunda afirmación del Teorema 2.

- Aplicando una transformación congruente con $\operatorname{diag}\left\{S^{-1}, I, I\right\}$ y complementos de Schur en la primera LMI de (17) se obtiene que $\mathcal{A} S^{-1} \mathcal{A}^{T}+\mathcal{G} V \mathcal{G}^{T} \preceq S^{-1}$. Comparando esta desigualdad con la matriz de covarianzas de régimen permanente $P_{0}$ de $(14)$, se tiene que $P_{0} \preceq S^{-1}$ si se asume que $\mathcal{A}$ tiene autovalores estables, lo cual ha sido demostrado en el primer punto. Entonces, tras aplicar complementos de Schur en la segunda LMI de (17) se obtiene $R S^{-1} R^{T} \preceq \bar{Q}$, que finalmente $d a$ lugar $a Q=R P_{0} R^{T} \preceq R S^{-1} R^{T} \preceq \bar{Q}$, lo que demuestra la tercera afirmación del Teorema 2.

Aprovechando las cotas presentadas en el teorema anterior, la siguiente estrategia muestra como diseñar el diagnosticador de fallos basado en BMI garantizando una FAR y un fallo mínimo detectable determinados, mientras se minimiza el ISE de los residuos.

Estrategia 2 Se define la matriz diagonal $\mathcal{Q}$ como en (15) con $\bar{\phi}(i)$ la FAR y $\bar{f}_{m}(i)$ el fallo minimo detectable deseados. El problema de optimización convexa

minimize $\gamma$

subject to $\mathcal{X}_{2}=\left\{\begin{array}{l}(17),(18), \\ \bar{Q} \preceq \mathcal{Q}, \sum_{i}^{n_{f}} \Gamma_{i}(i, i) \preceq \gamma, \\ \sum_{i, i \neq j}^{n_{f}} \Gamma_{i}(j, j) \preceq \epsilon \gamma, \forall i, j\end{array}\right\}$

a lo largo de las variables $S, \bar{Q}, P_{i}, y \Gamma_{i}$ con $\epsilon \geq 0$ $y \epsilon \rightarrow 0$, da lugar al diagnosticador de fallos con el mínimo valor de ISE debido a los fallos, y que garantiza el FAR y los fallos mínimos detectables requeridos, cuando se definen los umbrales de los evaluadores de residuos como $r_{t h}(i)=\bar{f}_{m}(i)$. Este diseño además permite reforzar el aislamiento dinámico de fallos a través del parámetro $\epsilon$.

Nota 4 Mientras que los elementos $\Gamma_{i}(i, i) \quad(i=$ $\left.1, \ldots, n_{f}\right)$ cuantifican la capacidad de seguimiento de un resudio $i$ respecto de un fallo en su propio canal, los elementos $\Gamma_{i}(j, j) \quad(i \neq j)$ representan su capacidad de rechazo de los fallos en un canal diferente, $f(j)$. Esto podría suponer un cierto compromiso entre capacidad de seguimiento y de desacoplamiento. Si el sistema es tal que se puede lograr un aislamiento dinámico de fallos completo, se puede fijar $\epsilon=0$ en (19); pero si eso no es posible debido a las características del sistema, se puede incluir la variable $\epsilon$ en el procedimiento de diseño basado en optimización. 
Nota 5 El problema de optimización anterior incluye BMIs y puede resolverse por medio de diversos algoritmos (solvers), como los presentados en [12,18]. Sin embargo, también es posible resolver ese problema de forma iterativa, a través de una secuencia de problemas LMI, siguiendo por ejemplo el enfoque de [8], o por medio de un único problema LMI usando algunas variables de relajación como por ejemplo en [10] con el inconveniente de aumentar el conservadurismo de la solución.

\section{EJEMPLO}

Considérese el sistema (1) descrito por las siguientes matrices

$$
\begin{gathered}
A=\left[\begin{array}{rr}
0,2883 & -0,0484 \\
-0,9076 & 0,7753
\end{array}\right], B=\left[\begin{array}{l}
0,0059 \\
1,1532
\end{array}\right], \\
C=\left[\begin{array}{rr}
-2,0756 & -0,1952 \\
-2,0756 & -0,1952
\end{array}\right]
\end{gathered}
$$

y $D=0$. Se supone la presencia de tres ruidos gaussianos no correlados de media cero y varianza 0.048. Esos ruidos afectan al sistema según las matrices

$$
G=\left[\begin{array}{lll}
0,0059 & 0 & 0 \\
1,1532 & 0 & 0
\end{array}\right], H=\left[\begin{array}{lll}
0 & 1 & 0 \\
0 & 0 & 1
\end{array}\right]
$$

Se supone que puede ocurrir un fallo en un actuador y en un sensor, es decir,

$$
E=\left[\begin{array}{rr}
-1,1114 & 0 \\
4,7135 & 0
\end{array}\right], F=\left[\begin{array}{ll}
0 & 0 \\
0 & 1
\end{array}\right] \text {. }
$$

Para este sistema LTI, se diseñan diagnosticadores de fallos que permitan reconocer fallos en el actuador y en un sensor por encima de $0.05 \mathrm{y}$ 0.10 respectivamente $\left(\bar{f}_{m}(1)=0,05\right.$ and $\bar{f}_{m}(2)=$ $0,10)$. Imponemos además que deberían transcurrir 250 muestras en promedio entre falsas alarmas $(\bar{\phi}(1)=\bar{\phi}(2)=0,004)$. La matriz de ganancias constante para la generación del residuo (4) obtenida mediante el método basado en Kalman es

$$
L^{T}=\left[\begin{array}{rrrr}
-0,0362 & 0,2285 & 0,0132 & -0,0127 \\
-0,0025 & -0,0700 & 0,0044 & 0,0325
\end{array}\right]
$$

mientras que si se diseña el observador con la técnica basada en BMIs, la matriz de ganancias constante es

$$
L^{T}=\left[\begin{array}{rrrr}
-0,4607 & 3,6390 & 0,0129 & -0,0227 \\
-0,0236 & 0,1001 & 0,0004 & 0,0227
\end{array}\right] \text {. }
$$

En este ejemplo, el problema se resolvió utilizando el solver PENBMI $[12,18]$ fijando, además, $\epsilon=0$.

Para verificar que se cumplen las restricciones de comportamiento impuestas, se simula en primer lugar un proceso sin fallos durante $3,5 \cdot 10^{6}$

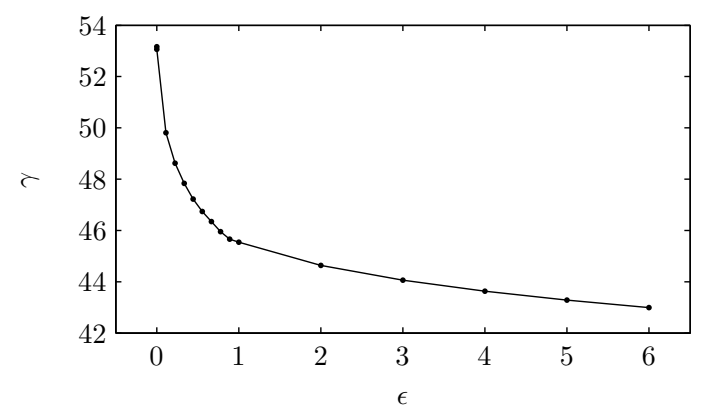

Figura 1: Compromiso entre restricción de aislamiento dinámico, $\epsilon$, y capacidad de minimización, $\gamma$

muestras. El detector basado en Kalman cumple las cotas de FAR impuestas $(\phi(1)=0,0040 \mathrm{y}$ $\phi(2)=0,0039)$; lo mismo sucede con el detector basado en BMI $(\phi(1)=0,0039$ y $\phi(2)=0,0039)$. En segundo lugar, se simula el proceso durante 3500 muestras en las que suceden los siguientes fallos

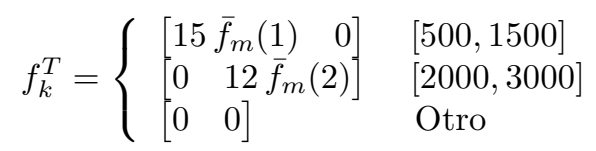

La Figura 2 muestra los resultados de simulación. El ISE asociado al fallo $f(1)$ verifica la cota obtenida en la optimización basada en BMI, es decir, $\varphi(1)<\Gamma_{1}(1,1) f(1)^{2}(16,96<17,25)$. Lo mismo sucede con el fallo $f(2)(26,87<29,28)$. Se comprueba además que, mientras el detector basado en BMI garantiza el aislamiento perfecto de los fallos, el detector basado en Kalman no logra el aislamiento dinámico de los fallos. Es importante notar que mejorar el aislamiento dinámico de los fallos implica reducir la capacidad de minimización, por lo que no se logra un ISE tan bajo frente a fallos escalón. La Figura 1 ilustra este compromiso para el caso de estudio, al mostrar el resultado de la optimización $\gamma$ para diferentes valores de $\epsilon$.

\section{CONCLUSIONES}

Se han propuesto dos enfoques para resolver el problema del diagnóstico de fallos con observadores PI basados en modelo bajo la suposición de ruidos gaussianos. El diseño se basa en el compromiso entre diversos índices de comportamiento de aislamiento, como la tasa de falsas alarmas (FAR), el fallo mínimo detectable y la capacidad de seguimiento medida mediante la integral del error al cuadrado (ISE). Se ha detallado cómo diseñar el diagnosticador de forma que se consiga un determinado valor de FAR y de fallo mínimo detectable a la vez que se minimiza el tiempo de respuesta a los fallos. Se han propuesto dos métodos de diseño 

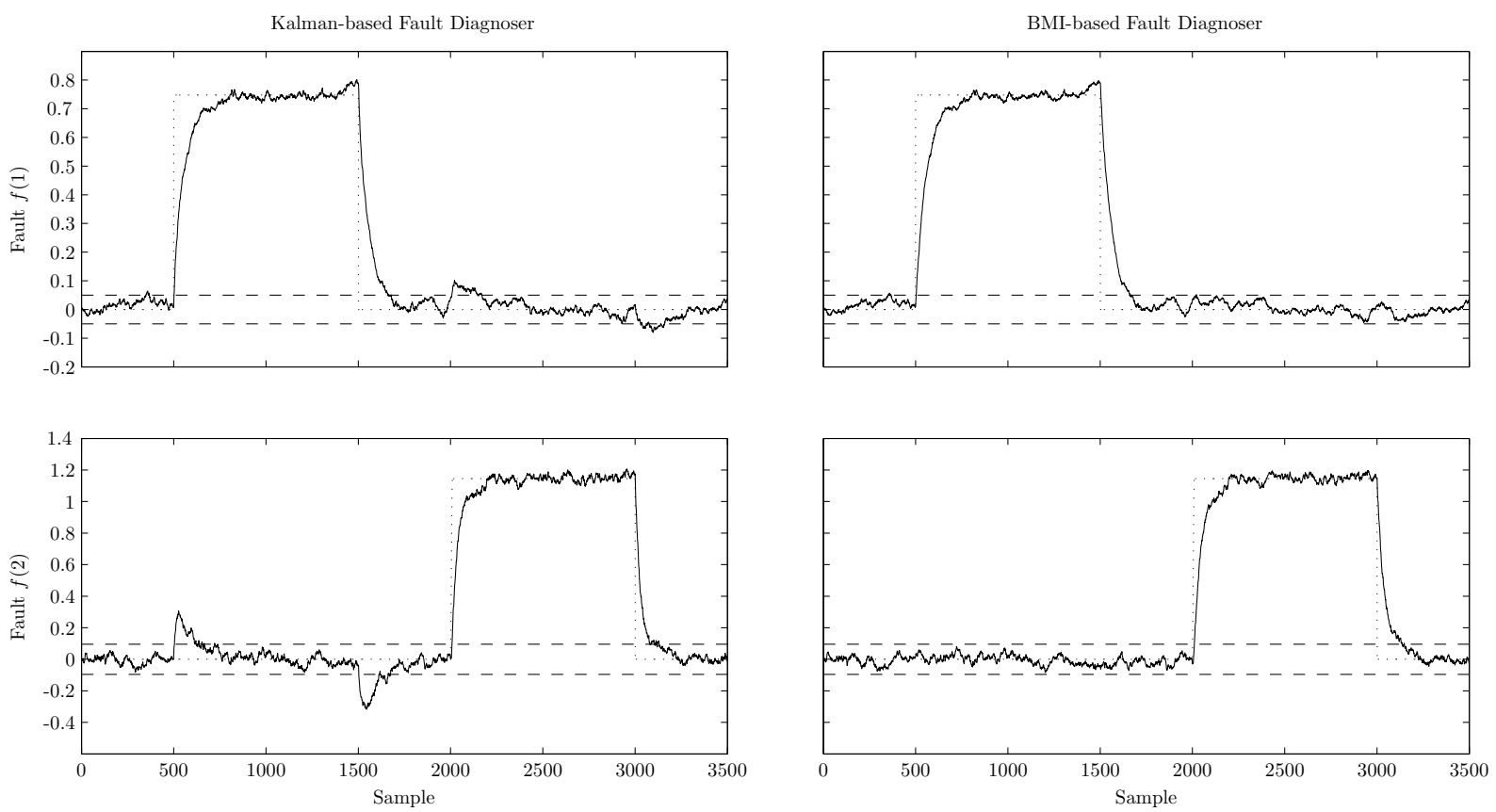

Figura 2: Diagnóstico de fallos con distintos enfoques. Fallo (. •), residuo (-), umbral (- -).

basado en procedimientos de optimización. El primero incluye como restricciones dos ecuaciones de Lyapunov discretas (filtro de Kalman de régimen permanente), mientras que el segundo incluye un conjunto de BMIs que acotan la matriz de covarianzas del error de estimación, y la integral del cuadrado del error ante fallos en escalón. En el enfoque basado en BMIs se puede tratar de evitar el acoplamiento dinámico entre estimaciones de fallos, logrando así un aislamiento dinámico de fallos, mientras que en el basado en filtro de Kalman no existe esa posibilidad. Mediante un ejemplo numérico se ha mostrado que con esta metodología se puede conseguir un determinado FAR mientras se optimiza el tiempo de respuesta ante fallos, a la vez que se garantiza un fallo mínimo detectable. El ejemplo también ha permitido ilustrar como el método basado en BMIs permite abordar además de forma explícita, el aislamiento dinámico de fallos.

\section{Agradecimientos}

Este trabajo ha sido financiado por la Universitat Jaume I de Castelló a través del proyecto con referencia P11B2015-42 y por el Ministerio de Economía y Competitividad a través del proyecto con referencia TEC2015-69155-R. También se ha financiado a través de la ayuda predocotoral FPU14/01592 del Ministerio de Educación, Cultura y Deporte y de la ayuda con referencia PI15734 de la Universitat Jaume I.

\section{Referencias}

[1] J. Chen and R.J. Patton. Robust modelbased fault diagnosis for dynamic systems, volume 3. Springer Science \& Business Media, 2012.

[2] J. Chen, R.J. Patton, and H. Zhang. Design of unknown input observers and robust fault detection filters. International Journal of Control, 63(1):85-105, 1996.

[3] M. Chilali and P. Gahinet. Hínf design with pole placement constraints: an LMI approach. IEEE Transactions on Automatic Control, 41(3):358-367, 1996.

[4] S.X. Ding. Model-based fault diagnosis techniques: design schemes, algorithms, and tools. Springer Science \& Business Media, 2008.

[5] S.X. Ding, T. Jeinsch, P.M. Frank, and E.L. Ding. A unified approach to the optimization of fault detection systems. International Journal of Adaptive Control and Signal Processing, 14(7):725-745, 2000.

[6] D. Dolz, I. Peñarrocha, and R. Sanchis. Performance trade-offs for networked jump observer-based fault diagnosis. IEEE Transactions on Signal Processing, 63(10):26922703, 2015.

[7] G. Duan and A. Wu. Robust fault detection in linear systems based on PI observers. International Journal of Systems Science, 37(12):809-816, 2006. 
[8] L. El Ghaoui, F. Oustry, and M. AitRami. A cone complementarity linearization algorithm for static output-feedback and related problems. IEEE Transactions on Automatic Control, 42(8):1171-1176, 1997.

[9] P.M. Frank, S.X. Ding, and T. Marcu. Modelbased fault diagnosis in technical processes. Transactions of the Institute of Measurement and Control, 22(1):57-101, 2000.

[10] J.C. Geromel, C.C. De Souza, and R.E. Skelton. LMI numerical solution for output feedback stabilization. In American Control Conference, 1994, volume 1, pages 40-44. IEEE, 1994.

[11] J. Guo, X. Huang, and Y. Cui. Design and analysis of robust fault detection filter using LMI tools. Computers \& Mathematics with Applications, 57(11):1743-1747, 2009.

[12] Di. Henrion, J. Löfberg, M. Kočvara, and M. Stingl. Solving polynomial static output feedback problems with penbmi. In 44th IEEE Conference on Decision and Control, 2005., pages 7581-7586. IEEE, 2005.

[13] R. Isermann. Model-based fault-detection and diagnosis-status and applications. $A n$ nual Reviews in control, 29(1):71-85, 2005.

[14] R. Isermann. Fault-diagnosis systems: an introduction from fault detection to fault tolerance. Springer Science \& Business Media, 2006.

[15] R. Isermann and P. Ballé. Trends in the application of model-based fault detection and diagnosis of technical processes. Control Engineering Practice, 5(5):709-719, 1997.

[16] R.E. Kalman. A new approach to linear filtering and prediction problems. Journal of Fluids Engineering, 82(1):35-45, 1960.

[17] A. Khedher, K. Benothman, D. Maquin, and M. Benrejeb. State and sensor faults estimation via a proportional integral observer. In 6th International Multi-Conference on Systems, Signals and Devices, 2009., pages 1-6. IEEE, 2009.

[18] M. Kočvara and M. Stingl. Pennon: A code for convex nonlinear and semidefinite programming. Optimization methods and software, 18(3):317-333, 2003.

[19] D. Koenig. Unknown input proportional multiple-integral observer design for linear descriptor systems: application to state and fault estimation. IEEE Transactions on Automatic Control, 50(2):212-217, 2005.
[20] B. Marx, D. Koenig, and D. Georges. Robust fault diagnosis for linear descriptor systems using proportional integral observers. In Proceedings. 42nd IEEE Conference on Decision and Control, 2003., volume 1, pages 457-462. IEEE, 2003.

[21] S. Montes de Oca, V. Puig, and J. Blesa. Robust fault detection based on adaptive threshold generation using interval LPV observers. International Journal of Adaptive Control and Signal Processing, 26(3):258283, 2012.

[22] M. Mrugalski. An unscented kalman filter in designing dynamic GMDH neural networks for robust fault detection. International Journal of Applied Mathematics and Computer Science, 23(1):157-169, 2013.

[23] J. Niemann, H.H.and Stoustrup, B. Shafai, and S. Beale. LTR design of proportionalintegral observers. International Journal of Robust and Nonlinear Control, 5(7):671-693, 1995.

[24] R.J. Patton and J. Chen. On eigenstructure assignment for robust fault diagnosis. International Journal of Robust and Nonlinear Control, 10(14):1193-1208, 2000.

[25] R.J. Patton, J. Chen, and J.H.P. Miller. A robust disturbance decoupling approach to fault detection in process systems. In Proceedings of the 30th IEEE Conference on Decision and Control, 1991., pages 1543-1548. IEEE, 1991.

[26] E. Sales-Setién, I. Peñarrocha, D. Dolz, and R. Sanchis. Fault detection in the blade and pitch system of a wind turbine with $\mathrm{H} 2 \mathrm{PI}$ observers. Journal of Physics: Conference Series, 659(1):012033, 2015.

[27] V. Venkatasubramanian, R. Rengaswamy, K. Yin, and S.N. Kavuri. A review of process fault detection and diagnosis: Part I: Quantitative model-based methods. Computers $\&$ Chemical Engineering, 27(3):293-311, 2003.

[28] P. Zhang and S.X. Ding. An integrated tradeoff design of observer based fault detection systems. Automatica, 44(7):1886-1894, 2008.

[29] M. Zhong, S.X. Ding, and E.L. Ding. Optimal fault detection for linear discrete timevarying systems. Automatica, 46(8):13951400, 2010.

[30] M. Zhong, S.X. Ding, J. Lam, and H. Wang. An LMI approach to design robust fault detection filter for uncertain LTI systems. Automatica, 39(3):543-550, 2003. 